\title{
Anethole Stability in Aniseed Spirits: Storage Condition Repercussions on Commercial Products
}

\author{
Veronica Vendramin ${ }^{1}\left(\mathbb{D}\right.$, Antonio Pesce ${ }^{2}$ and Simone Vincenzi ${ }^{1, *}$ \\ 1 Department of Agronomy, Food, Natural Resources, Animals and Environment (DAFNAE), \\ University of Padova, Viale dell'Università 16, 35020 Legnaro, Italy; veronica.vendramin@unipd.it \\ 2 Centre for Research in Viticulture and Enology (CIRVE), University of Padova, Viale XXVIII Aprile 14, \\ 31015 Conegliano, Italy; pesce.antonio.88@gmail.com \\ * Correspondence: simone.vincenzi@unipd.it
}

check for updates

Citation: Vendramin, V.; Pesce, A.; Vincenzi, S. Anethole Stability in Aniseed Spirits: Storage Condition Repercussions on Commercial Products. Beverages 2021, 7, 73. https://doi.org/10.3390/ beverages7040073

Academic Editor: Pavel Dostálek

Received: 4 October 2021

Accepted: 26 October 2021

Published: 30 October 2021

Publisher's Note: MDPI stays neutral with regard to jurisdictional claims in published maps and institutional affiliations.

Copyright: (c) 2021 by the authors. Licensee MDPI, Basel, Switzerland. This article is an open access article distributed under the terms and conditions of the Creative Commons Attribution (CC BY) license (https:// creativecommons.org/licenses/by/ $4.0 /)$.

\begin{abstract}
Background: trans-anethole is the main component of anise, and its flavor is characteristic of all the aniseed spirits. It is known that trans-anethole oil/ethanol/water solution could result in the undesirable emulsion effect called the "ouzo effect". Moreover, trans-anethole can undergo chemical decay, producing unhealthy compounds, mainly cis-anethole and para-anisaldehyde. In this work, the trans-anethole stability was challenged simulating commercial products' long-term storage. Methods: Anethole standard solutions and two Italian aniseed spirits, namely Sambuca and Mistrà, were used to evaluate the trans-anethole response to light (both ultraviolet and visible) and its stability under thermal stress. Anethole decay was monitored by trans-anethole quantification using an HPLC method. Results: Light and high-temperature exposure affect the trans-anethole content of aniseed spirits during the storage, but sucrose revealed a role in the trans-anethole protection. Conclusions: as transport and storage are involved in the anethole decay, the Regulations on the minimum of the anethole that must be a guarantee in the aniseed spirits should be revised.
\end{abstract}

Keywords: trans-anethole; aniseed spirits; light stress; thermal stress; storage stability

\section{Introduction}

trans-anethole (1-methoxy-4-[(E)-prop-1-enyl]benzene, $t$-ANE) is one of the main constituents of numerous essential oils such as anise, clove, cinnamon, fennel, and thyme [1,2] and the principal volatile compound of aniseed spirits [3]. $t$-ANE was introduced to replace absinthe when it was made illegal in the late 1800s, and today, it is widely used in the food and spirit industries [1]. In Mediterranean countries, aniseed spirits include, for example, Pastis, Pernod, Ricard (in France), Ouzo (in Greece), Raki (in Turkey), Mistrà, and Sambuca (in Italy). In the past years, aniseed spirits stability was studied with particular attention to the "ouzo effect", namely the physicochemical equilibrium that permits the $t$-ANE oil drops dissolution into water/ethanol solution, avoiding emulsion formation [4,5]. Besides emulsification, aniseed spirits are affected by other organoleptic alterations because $t$-ANE could undergo chemical decay processes. Three main mechanisms were identified, namely isomerization, dimerization, and oxidation $[2,6]$. In isomerization, triplet anethole is generated by electron transfer, and its decay produces a mixture of cis and trans isomers. Dimerization occurs via cycloaddition of a cation radical to a neutral molecule and results in dimers formation. Cycloaddition could involve either two molecules of one chemical species or molecules belonging to two different species. Instead, oxidation is the reaction of cation radical monomers with $\mathrm{O} 2$ [7]. Considering their formation rate, the main $t$-ANEderived products are cis-anethole ( $c$-ANE) from isomerization, para-anisaldehyde ( $p$-ANI) from oxidation, and photoanethole ( $p h$-ANE) from cycloaddition between $t$-ANE and p-ANI. $t$-ANE demonstrated several health-promoting functions at low concentrations, i.e., anti-inflammatory, pro-apoptotic, anti-metastatic, and neuroprotective [8], however at high doses, its metabolites show undesirable effects. In human volunteers, the administration of 
carbon-labeled molecules revealed that $t$-ANE is metabolized principally by oxidation and demethylation, while $3 \%$ was transformed to anethole $1^{\prime}, 2^{\prime}$-epoxide, which is reputed hepatotoxic [9]. The cis isomer, $c$-ANE, is considered potentially toxic for humans because the hydrolysis rate of its epoxide was demonstrated to be 50 times slower than those of $t$-ANE epoxide [10]. Moreover, $p$-ANI was recognized to stimulate dermatitis [11], and $p h$-ANE is supposed to have estrogenic effects [12]. $c$-ANE and $p$-ANI are commonly present in aniseed spirits at low concentrations [3], while there is no evidence of the $p h$-ANE presence in beverages to date. In the attempt to increase $t$-ANE stability over the product shelf-life, several stabilizers were evaluated with the aim to prolong $t$-ANE maintenance in foods, i.e., gelatin gels, starch matrices, and cyclodextrin above all [13,14], but this solution could not be considered suitable for spirits. Nevertheless, sucrose, which is present in many aniseed spirit recipes, revealed interesting aroma retention properties, which were attributed to its viscosity and molecular affinity to high volatile flavors [15]. Ultraviolet (UV) and visible (VIS) lights, temperature, and atmospheric oxygen are known to influence the $t$-anethole chemical stability [8]. It should be taken into account that those factors can be all present during the prolonged storage of liquors. UV and VIS lights are considered accelerating autoxidation processes. Fennel oil maintained in dark conditions underwent the same alterations recognized under light storage, but they showed results considerably slower [16]. Similarly, anise essential oil irradiated by sunlight or by UV lights developed a high value of new former $p h$-ANE [17]. In sweet fennel oil, $t$-ANE was completely oxidized to ANI or isomerized to $c$-ANE after 2 months of storage under light at room temperature [16]. Additionally, $t$-ANE was converted into $c$-ANE when treated with UV rays or high temperatures [18]. Storage temperature also strongly influences essential oil stability. Chemical reactions accelerate with temperature increase due to the temperature-dependence of the reaction rate. On the contrary, low temperatures favor the solubility of oxygen in liquids, which negatively affect essential oil stability. In fact, Btaun and Franz [19] highlighted that stability is reduced by prolonged storage time as well as the temperature rise and that the most important change recorded by the comparison of fennel oil storage at 25 and at $4{ }^{\circ} \mathrm{C}$ was the rapid increase in $p$-ANI in the former. Storage condition effects on aniseed liquor were explored by Grillo [5], combining increasing temperatures (up to $35^{\circ} \mathrm{C}$ ) and light exposure on typical French aniseed spirits (Pastis) evaluating, however only its emulsion stability. The study of the degradation of $t$-ANE in aniseed spirits is important not only for eventual health problems due to the appearance of dangerous side products but also from the legislative point of view. In fact, the $t$-ANE content in aniseed spirits, at least in Europe "Regulation (EU) 2019/787," 2019, should respect a minimum value of concentration (generally between 1 and $2 \mathrm{~g} / \mathrm{L}$ ) and the natural decrease in its content due to incorrect storage could result in legal actions against the producers. In the present study, $t$-ANE stability in two Italian aniseed spirits was evaluated with particular attention to $t$-ANE chemical degradation. Commercial Sambuca and Mistrà spirits were subjected to UV-VIS light and temperature stress. The role of sucrose (present at high concentration in Sambuca) in $t$-ANE photo and thermal protection was explored using additional model solutions.

\section{Materials and Methods}

\subsection{Samples and Solutions}

trans-anethole ( $t$-ANE) was determined in two commercially available Italian aniseed drinks, namely Mistrà and Sambuca. According to Regulation (EU) 2019/787 [20], Mistrà contains $40 \%$ ethanol and $0 \mathrm{~g} / \mathrm{L}$ of sugars, and Sambuca contains $40 \%$ ethanol and $350 \mathrm{~g} / \mathrm{L}$ of sugars. For Sambuca (freshly prepared), the content of $t$-ANE was around $1.2 \mathrm{~g} / \mathrm{L}$, while in Mistrà (11 months old sample, stored in the warehouse of the producer), it was under the legal limit and corresponded to $0.63 \mathrm{~g} / \mathrm{L}$. The lower content of $t$-ANE in Mistrà could be explained by a degradation that occurred during the storage, as explained in the following sections. In order to evaluate the effect of sugar on $t$-ANE, four model solutions containing 
$40 \%$ ethanol and $0,125,250$, and $350 \mathrm{~g} / \mathrm{L}$ of sucrose were used during the tests. All the solutions were added with $1 \mathrm{~g} / \mathrm{L}$ of standard $t$-ANE (Sigma, Milan, Italy).

\subsection{High-Pressure Liquid Chromatography (HPLC) and Gas Chromatography (GC) Methods}

For $t$-ANE quantification, the HPLC method proposed by Jurado and colleagues [21] was modified and validated by comparison with the official GC method. The chromatographic system used was a Waters system (1525 Binary Pump) provided with a UV detector (Waters 2487 Dual Band Absorbance Detector) and autosampler (Waters 2707) controlled with Software Breeze Version 3.3 (Waters S.p.A., Milan, Italy). Compounds were separated by C18 Kinetex ( $4.6 \mathrm{~mm} \times 150 \mathrm{~mm}, 5 \mu \mathrm{m}$, Phenomenex) in isocratic elution with methanolwater (65:35) mobile phase and a flow rate of $1 \mathrm{~mL} / \mathrm{min}$. The column compartment was maintained at $37^{\circ} \mathrm{C}$, and $10 \mu \mathrm{L}$ of samples were injected using full loop injection. The detection was performed at $257 \mathrm{~nm}$, and an external calibration curve $(0.0125-0.2 \mathrm{~g} / \mathrm{L}$ for all compounds) was used for the identification and quantification of selected compounds. Standard solution of $t$-ANE (99\%), $p$-anisaldehyde ( $p$-ANI, $\geq 97.5 \%)$, and $p$-allylanisole ( $p$-ALL, $\geq 98 \%$ ) analytical grade were purchased from Sigma-Aldrich (Milan, Italy). Absolute ethanol and methanol HPLC grade were supplied by VWR International S.r.l. (Milan, Italy). Before injection, samples were diluted 10 times into 50\% $v / v$ methanol-water and filtered using a $0.45 \mu \mathrm{m}$ cellulose acetate filter (Millipore, Milan, Italy) before the injection.

GC analyses were performed following the European regulation [22] using a GC-2025 (Shimadzu, Milan, Italy) equipped with an HP Innowax capillary column $(25 \mathrm{~m}, 0.25 \mathrm{~mm}$ internal diameter, $0.25 \mu \mathrm{m}$ film, Agilent Technologies, Milan Italy) with helium at $2 \mathrm{~mL} / \mathrm{min}$ as the carrier gas. The injector temperature was $250{ }^{\circ} \mathrm{C}$, while the separation was performed in isothermal conditions at $180^{\circ} \mathrm{C}$. The system was provided with autosampler AOC-20i and FID detector 2010 Plus (Shimadzu, Milan, Italy). Before the injection, Sambuca samples were processed by saturation with anhydrous ammonium phosphate and sodium bibasic phosphate in order to induce the separation of the alcoholic phase and eliminate sugars from the sample. The injection volume was $1 \mu \mathrm{L}$ with split 1:40. The $t$-anethole peak was identified by the comparison with an external standard. Additionally, an internal standard ( $p$-ALL, $0.2 \mathrm{~g} / \mathrm{L}$ final concentration) was used as an internal standard to verify sample extraction yield.

\subsection{Ultraviolet (UV) and Visible (VIS) Lights Exposure Assay}

Samples were exposed to UV transilluminator TFX (Euroclone, Milan, Italy). Two wavelengths were chosen, namely 254 (UVC) and 312 (UVB) nm corresponding to a radiant intensity of $7 \mathrm{~mW} / \mathrm{cm}^{2}$ and $8 \mathrm{~mW} / \mathrm{cm}^{2}$, respectively. For the VIS treatment, light 7 stress (EVER, Pramaggiore, Italy) was used. This instrument consists of a box with different light-emitting diodes (LED), which could be used singularly or in combination. This system was chosen for the possibility of modulating the color of light during exposure. Samples (four replicates) were transferred into a $10 \mathrm{~mL}$ Pyrex test tube and kept perpendicularly to the light bulbs in order to maximize the exposure surface for $20 \mathrm{~min}$. This time has been chosen based on preliminary results, as it was enough to observe t-anethol degradation, but at the same time not long enough to cause a significant increase in temperature. Model solutions were treated as described before in the UV assay in order to verify the role of sucrose in photo-protection. Samples were then processed by HPLC. When necessary, peak identity was confirmed by comparison with the official GC method.

\subsection{High-Temperature Assay}

Both samples (Mistrà, Sambuca) and model solutions were used to explore the $t$ anethole stability under high-temperature stress. Four samples $(10 \mathrm{~mL})$ for each solution were kept for $5 \mathrm{~h}$ either at 40,50 , or $60^{\circ} \mathrm{C}$ in a water bath (Elettrofor, Rovigo, Italy) using the same Pyrex tube used for the light stress experiments. trans-anethole was quantified by HPLC. 


\subsection{Low-Temperature Assay}

Mistrà, Sambuca, and the four model solutions were used for the low-temperature storage test. Each sample was divided into four $10 \mathrm{~mL}$ Pyrex glass tubes and kept in dark conditions at the constant temperature of $4{ }^{\circ} \mathrm{C}$ for 3 months. At the end of the incubation period, the formation of precipitates was analyzed visually in all the samples.

\subsection{Statistical Analyses}

Mean values and standard deviation were used to express the results. Data were subjected to analysis of variance to determine significant differences between the samples $(p<0.01)$ by ANOVA, and differences between the treatment means were distinguished using Tukey's HSD test. All the analyses have been performed by XLSTAT ver. 2016.02.29253 (Addinsoft, Long Island City, NY, USA).

\section{Results and Discussion}

\subsection{HPLC Method Validation}

European regulation requires the usage of the GC method for the trans-anethole ( $t$ ANE) quantification in spirits [22]. In the presence of high sugar concentration, like in many aniseed spirits, however, a step allowing for sugar separation is necessary before GC injection. In 2006, Jurado et al. [21] proposed a new protocol, based on the HPLC technique, as an alternative for $t$-ANE quantification in beverages characterized by high sugar concentration. This method allows a significant reduction in terms of time and costs, bypassing the sample extraction. In the present work, the HPLC method has been slightly modified and adapted to the Kinetex column changing the proportion of the elution phase into 65:35 methanol/water and increasing the column temperature up to $37^{\circ} \mathrm{C}$ in order to favor method repeatability. This method was validated by comparing HPLC quantification with results obtained from the GC analyses. Two commercial spirits (Mistrà and Sambuca) and a model solution prepared with $350 \mathrm{~g} / \mathrm{L}$ of sucrose and $1 \mathrm{~g} / \mathrm{L}$ of $t$-ANE were used. Three compounds were selected for the methods comparison, i.e., $t$-ANE, $p$-allylanisole ( $p$-ALL), used as internal standard during sample extraction, and p-anisaldehyde ( $p$-ANI), which represent one of the principal oxidation products of $t$-ANE [7]. As suggested before [21], the wavelength chosen for the analyses was $257 \mathrm{~nm}$. The HPLC chromatogram reveals three well-defined peaks corresponding to $p$-ANI, $p$-ALL, and $t$-ANE, respectively (Figure 1). Their retention times were 2.2, 5.3, and 5.9 min (Figure 1).

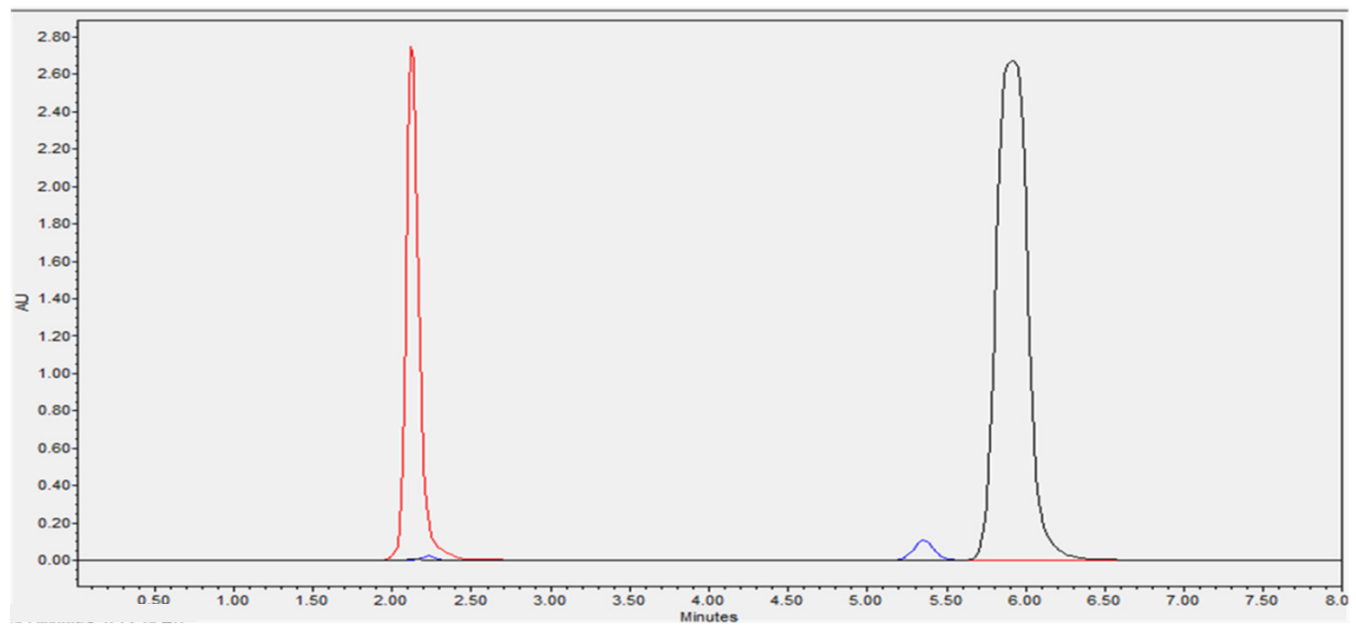

Figure 1. HPLC chromatogram of standard solutions. (1) $p$-anisaldehyde, RT $=2.2 \mathrm{~min}$, (2) $p$ allylanisole, $\mathrm{RT}=5.3 \mathrm{~min}$, and (3) $t$-anethole, $\mathrm{RT}=5.9 \mathrm{~min}$, peaks are shown.

t-ANE quantification of commercial spirits and model solutions obtained by HPLC and GC methods were compared using the ANOVA test. Statistical analysis evidenced no 
significant difference between HPLC and GC quantifications for all the three samples, as reported in Table 1.

Table 1. Analytic methods comparison on $t$-anethole quantification. Three water/ethanol solutions (two commercial spirits, Sambuca and Mistrà and a model solution) were used. Means and standard deviations of two $\left(^{*}\right)$ and eight $\left({ }^{* *}\right)$ replications are expressed.

\begin{tabular}{cccccc}
\hline \multicolumn{2}{c}{ Sambuca } & \multicolumn{2}{c}{ Mistrà } & \multicolumn{2}{c}{ Model Solution } \\
\hline GC & HPLC & GC & HPLC & GC & HPLC \\
\hline $1.11 \pm 0.001^{*}$ & $1.18 \pm 0.071^{*}$ & $0.66 \pm 0.078^{*}$ & $0.63 \pm 0.042^{*}$ & $0.87 \pm 0.036^{* *}$ & $0.90 \pm 0.031^{* *}$ \\
\hline
\end{tabular}

Moreover, the error was estimated as variation (in percentage) on the pair-wise comparison of $t$-ANE quantification obtained by the GC and HPLC method. Taking into account 12 comparisons, the HPLC method revealed an average variation of $5.2 \%$, demonstrating greater accuracy with respect to Jurado and colleagues [21], which obtained an $8.8 \%$ average variation over 29 samples. $t$-ANE limit of detection (LOD) and limit of quantification (LOQ) have been calculated as the concentration of the mean blank signal plus three and ten standard deviations, respectively, according to Moretón-Lamas et al. [23] and Eurachem guide [24]. LOD correspond to $4.6 \mathrm{mg} / \mathrm{L}$ and LOQ to $12.7 \mathrm{mg} / \mathrm{L}$.

\subsection{Effect of UV Exposure}

With the aim to explore the role of UV irradiation during aniseed spirit storage on $t$-ANE degradation, it was chosen to expose samples to both UVC and UVB lights. Light stress was administered for $20 \mathrm{~min}$ at a UVC wavelength of $254 \mathrm{~nm}$ or UVB wavelength of $312 \mathrm{~nm}$. Samples were contained in clear Pyrex tubes during the treatment in order to simulate a clear bottle light transmission [25]. The experimental data revealed that both wavelengths induce the degradation of $t$-ANE, represented in Figure 2 by the decrease in the peak at $5.9 \mathrm{~min}$ and the appearance of a second peak at $5.7 \mathrm{~min}$, corresponding probably to a $t$-ANE degradation product. Unexpectedly, spirits revealed differences in the UV response. The major degradation effect was recorded for Mistrà, where the $312 \mathrm{~nm}$ light treatment reduced of about $40 \%$ the amount of $t$-ANE, while a reduction of about $30 \%$ was obtained upon $254 \mathrm{~nm}$ treatment (Figure 2). Nonetheless, in Mistrà, the comparison between $t$-ANE degradation at UVC and UVB wavelengths evidenced no statistically significant difference, while a statistically significant effect was registered for Sambuca. In fact, the $254 \mathrm{~nm}$ wavelength treatment degraded less than $20 \%$ of $t$-ANE, while anethole reduction was around $30 \%$ after $312 \mathrm{~nm}$ light exposure (Figure 2). Anethole has two peaks of absorption with a maximum at 254 and $258 \mathrm{~nm}$ in the two isoforms, cis and trans, respectively [26]. Therefore, a greater degradation (i.e., higher energy absorption) was expected at $254 \mathrm{~nm}$. In this work, however, UVB radiations seemed to affect more strongly $t$-ANE degradation. This effect is probably due to Pyrex glass, which has a sensible higher transmission in the UVB bands than in the UVC [27].

Besides determining $t$-ANE degradation, the formation of its side products was explored. Sample treatment at $312 \mathrm{~nm}$ induced the raising of $2.2 \mathrm{~min}$ peak in HPLC, and, as expected, peak increased more strongly in Mistrà sample. Firstly, the correspondence of RT time suggested that peak identifies $p$-ANI, which is one of the main by-products of $t$-ANE photo-oxidation [6]. Misharina and Polshkov [16] evidenced the progressive appearance of $p$-ANI and c-ANE during fennel oil light irradiation, along with $t$-ANE reduction. To confirm its identity, the official GC method was used, but it revealed the absence of a peak at $5 \mathrm{~min}$ ( $p$-ANI retention time in GC), contradicting the previous idea of $p$-ANI formation. Additionally, GC analyses revealed a new peak eluting at $2.8 \mathrm{~min}$, very close to the $t$-ANE retention time. Castro et al. [2] studied the GC profile characterizing pure solution of t-ANE in toluene after UV exposure and verified the formation of a new peak immediately prior to t-ANE elution. Authors recognized c-ANE as responsible for that peak. Similarly, the peak identified in this work at $2.8 \mathrm{~min}$ may originate from the isomerization of $t$-anethole too. 
c-ANE, besides having an unpleasant pungent odor, showed to be about 15-18 more toxic than $t$-ANE [8]. In the present work, the peak tentatively attributed to $c$-ANE represented only a marginal by-product of $t$-ANE degradation, so it should be considered not dangerous for consumer safety.

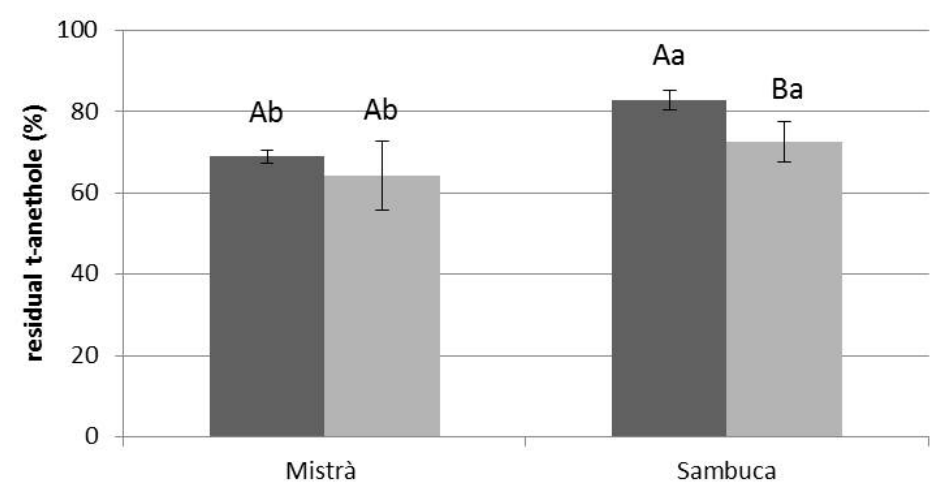

Figure 2. UV treatment at $254 \mathrm{~nm}$ (dark gray) and 312 nm (light gray) effects on Sambuca and Mistrà. Means and standard deviations of four replicates are reported. Different capital letters identify statistically significant $(p<0.01)$ differences between UV treatments, while lowercase letters identify differences between samples.

\subsection{Effect of UV Exposure on Model Solutions}

Sucrose solution could interfere with the light photo-degradation process in the UV range, where sucrose shows consistent light absorption. In fact, it was demonstrated that sucrose solution absorbance depends on sucrose concentration and light wavelength, with a sensible higher value at $254 \mathrm{~nm}$ than at $312 \mathrm{~nm}$ [28]. As Sambuca and Mistrà differ only for sugar content, it can be supposed that the different masking effects on $t$-ANE degradation registered in the aniseed spirits UV test could directly depend on the sucrose concentration. In order to explore this possibility, four model solutions (MS) containing $0,125,250$, and $350 \mathrm{~g} / \mathrm{L}$ of sucrose were exposed to UV stress. The effect of the sugar present in the solution was evident (Figure 3). Even in this case, results confirmed that the samples were significantly more affected by the UVB treatment; in this condition, $t$-ANE achieved about $45 \%$ of reduction in the absence of sugar while about $35 \%$ at the higher sugar concentration (Figure 3). Interestingly, a significant difference between samples with a lower amount of sugars (i.e., 0 and $125 \mathrm{~g} / \mathrm{L}$ ) and higher sugar content (i.e., 250 and $350 \mathrm{~g} / \mathrm{L}$ ) was detected. This demonstrated an evident photo-protection effect of sucrose. Contrarily, the analyses of model solutions after $254 \mathrm{~nm}$ treatment revealed a not linear relationship between photo-protection intensity and sucrose content.

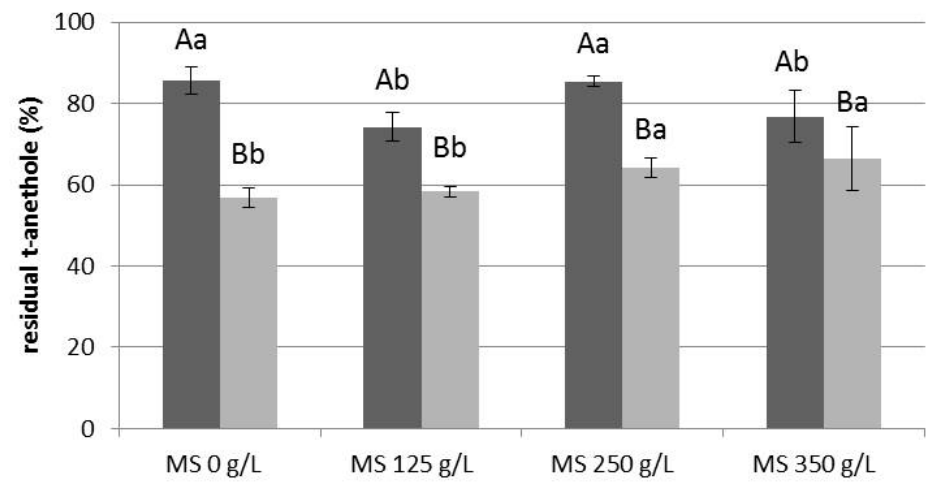

Figure 3. UV treatment at $254 \mathrm{~nm}$ (dark gray) and $312 \mathrm{~nm}$ (light gray) effects on model solutions $t$-anethole content. Means and standard deviations of four replicates are expressed. Different capital letters identify statistically significant $(p<0.01)$ differences between UV treatments, while lowercase letters identify differences between samples. 


\subsection{Effect of VIS Light Exposure}

Visible light exposure is one of the principal stresses for the commercial aniseed spirits during the market storage due to the limited filtering capacity of clear bottles against 400-800 nm wavelengths [25]. For this reason, $t$-ANE response to VIS light stress was taken into account, subjecting Sambuca samples to different color lights. The used system allowed to test three primary light colors (i.e., red, green, and blue, generated by LEDs) separately or in combination, obtaining three secondary colors (i.e., yellow, magenta, and cyan resulting from the primary LED lays overlap) and white, as the union of all the three primary LEDs.

This experiment reveals that all the LEDs caused a partial degradation of $t$-ANE, which, however, never achieved degradation levels recorded upon UV treatments (Figure 4). It should be kept into account that in the VIS light range, sucrose shows very low absorption capability, even though its absorbance increases between 400 and $500 \mathrm{~nm}$, achieving a plateau at $500 \mathrm{~nm}$ [28], so the observed differences at different wavelengths cannot be attributed to the presence of sucrose. In two out of seven treatments, namely yellow and cyan lights, residual $t$-ANE was significantly less (Figure 4). This is probably due to the combination of the high-energy green radiation (of 500-550 nm wavelengths) with the overlapping of second radiation, red or blue for yellow and cyan lights, respectively. In fact, magenta-treated samples, which were exposed only to blue and red lights together, show a $t$-ANE reduction similar to those recorded for single-ray treated samples. Unexpectedly, white light, which is derived from the overlap of all the primary color rays, shows a $t$-ANE reduction comparable to the singular primary colors.

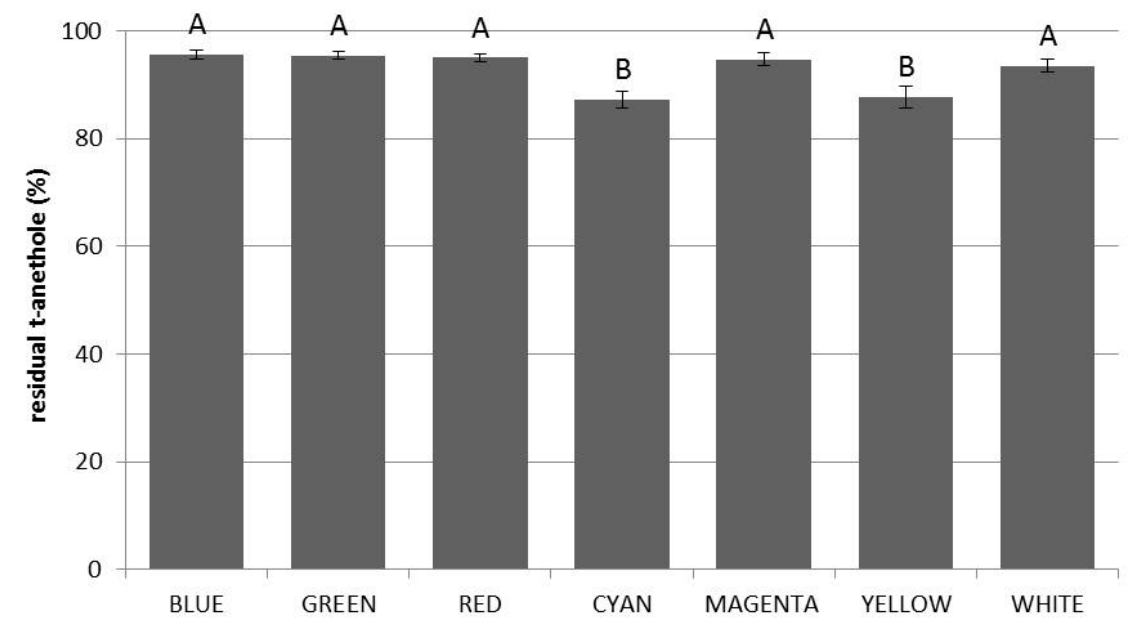

Figure 4. VIS light effects on Sambuca $t$-anethole reduction. Means and standard deviations of four replicates are expressed. Different letters identify statistically significant $(p<0.01)$ differences between treatments.

\subsection{High-Temperature Stability of Trans-Anethole}

The temperature recommended during aniseed spirits transport and storage is room temperature, but these entail important seasonal variations, for example, during transport. Nevertheless, little is known about $t$-ANE ethanol/water mixture stability under high temperatures [5]. In the present study, the $t$-ANE stability of Sambuca and Mistrà were verified in the extreme heat test. Samples were heated up to 40,50 , and $60^{\circ} \mathrm{C}$ for $5 \mathrm{~h}$.

As expected, the increase in temperature raised the amount of $t$-ANE undergoing degradation. This trend appears in both the commercial spirits (Figure 5a). Hightemperature treatment affects more strongly Mistrà in all the conditions, causing the maximum loss of $t$-ANE (about $60 \%$ of the original amount) in the most extreme condition. As for UV light stress, the difference of stability between spirits suggests an effect of sucrose on the $t$-ANE degradation rate. Therefore, this hypothesis was deeper explored using four $t$-ANE model solutions differing in sucrose concentration. 

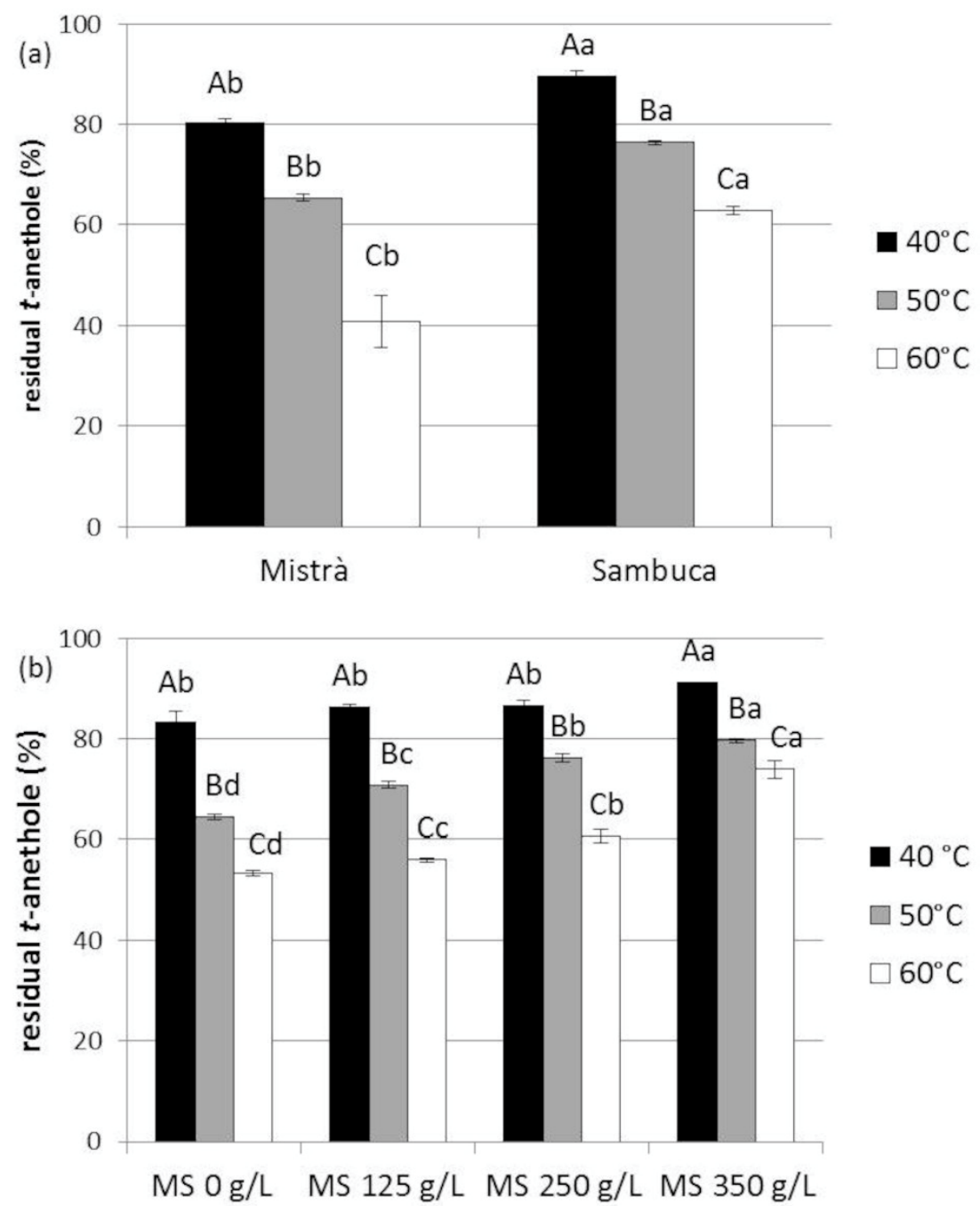

Figure 5. $t$-anethole quantification after thermal stress. (a): thermal effects on Sambuca and Mistrà. (b): thermal effects on model solutions. Means and standard deviations of four replicates are expressed. Different capital letters identify statistically significant $(p<0.01)$ differences between treatments, while different lowercase letters identify differences between samples.

This experiment confirmed the protecting effect of sugar at all the temperature levels (Figure 5b). The effect is magnified at higher temperatures, determining at $60{ }^{\circ} \mathrm{C}$ only about $25 \%$ of $t$-ANE loss at $350 \mathrm{~g} / \mathrm{L}$ respect to almost $50 \%$ of degradation in $0 \mathrm{~g} / \mathrm{L}$ sucrose solution (Figure $5 \mathrm{~b}$ ), while at $40{ }^{\circ} \mathrm{C}$ the losses were $10 \%$ and $18 \%$, respectively.

For the research purpose, it is important to establish if thermal degradation could represent a risk for the final consumer, leading to the formation of toxic compounds. Therefore both $p$-ANI and c-ANE were specifically analyzed. Contrary to Alvarez et al. [29], here, the thermal treatment did not result in a $p$-ANI increase. In neither HPLC nor GC profile, there was evidence of $p$-ANI formation. Similarly, even if it is well known that the increase in temperature enhances $t$-ANE isomerization [2], no evidence of an increase in the GC peak tentatively identified as c-ANE was recorded. To our knowledge, this is the first report on the role of sucrose in $t$-ANE preservation.

\subsection{Low-Temperature Stability of Trans-Anethole}

The aniseed spirits are considered "stable" products, and therefore, they are not subjected to particular care during their transport and storage. This means that liquors can be exposed, mainly in cold countries, to low temperatures even below $0{ }^{\circ} \mathrm{C}$. 
At low temperatures, a physical phenomenon can occur, which is $t$-ANE precipitation. As a matter of fact, the pure molecules of $t$-ANE show a melting point between 20 and $21^{\circ} \mathrm{C}$, which means that below this temperature, it appears as a solid. In aniseed spirits, the presence of ethanol definitely increases the $t$-ANE solubility. Previously, Sitnikova et al. [4] recorded spontaneous emulsification of water/ethanol/t-ANE oil when the temperature was lowered to $3{ }^{\circ} \mathrm{C}$, but the ethanol concentration was lower than $40 \%$, the concentration normally found in aniseed spirits. Indeed, precipitation phenomena were never reported in this kind of product, even after storage at low temperatures.

In this work, the effect of low-temperature exposure on $t$-ANE stability was verified by storing the samples of Sambuca, Mistrà, and the four model solutions for up to three months at $4{ }^{\circ} \mathrm{C}$ in the dark. Even though no effect on the $t$-ANE concentration was observed in both the aniseed spirits and model solutions (data not shown), the long-term storage of the latter led to $t$-ANE crystallization, even in the presence of $40 \%$ ethanol. This precipitation appeared only in the sample without sugars, while the solutions remained limpid when the concentrations of sucrose were higher or equal to $125 \mathrm{~g} / \mathrm{L}$. This behavior further supports the positive effect of sugars on $t$-ANE stability and suggests a major risk of its precipitation in Mistrà.

As reported above, however, in Mistrà samples, no crystal formation was noticed after the three months at $4{ }^{\circ} \mathrm{C}$. This could depend on the recipe of Mistrà (and Sambuca), which includes the addition of low quantities $(0.2 \%-0.3 \%)$ of a mixture of essential oils, which could be responsible for the subtle differences between liquor and model solution as well as among different commercial products.

In any case, even if $t$-ANE precipitation could occur at low temperatures, this does not represent a quality defect in the aniseed spirits, as the precipitation phenomenon has proved to be completely reversible when restoring room temperature.

\section{Conclusions}

Commercial aniseed spirits revealed $t$-anethole degradation under light and temperature stresses. The exposure of Sambuca and Mistrà to UV wavelengths determined stronger $t$-ANE degradation in Mistrà both at 254 and $312 \mathrm{~nm}$. Sambuca sample evidenced a low susceptibility to VIS light, and only for yellow and cyan lights. Finally, for the first time, the effect of relatively high temperatures $\left(40-60^{\circ} \mathrm{C}\right)$ on $t$-ANE degradation in the aniseed spirits was determined. A protection activity of sucrose against photo- and thermal degradations of $t$-ANE was proved and confirmed in model solutions containing ethanol/sucrose mixture.

This study, for the first time, demonstrates a possible degradation of $t$-ANE in aniseed spirits during transport and storage, with a significant degradation occurring even after short-term light or thermal treatments, and suggests that the Regulations of the minimum $t$-ANE content in aniseed spirits should be revised. In addition, it provides the producers a better knowledge of the stability of their products, highlighting that aniseed spirits without sugars are more susceptible to $t$-ANE degradation and, consequently, need more attention during preparation and in the packaging choice.

Author Contributions: S.V. conceived this study, supervised the work, and assisted with the final editing of the manuscript. A.P. assisted in the laboratory analyses and performed the sample collection, data curation with figure manipulation. V.V. wrote the original manuscript, assisted data curation and figure manipulation, and performed statistics. All authors have read and agreed to the published version of the manuscript.

Funding: This research received no external funding.

Institutional Review Board Statement: Not applicable.

Informed Consent Statement: Informed consent was obtained from all subjects involved in the study.

Data Availability Statement: Full data set available upon request to authors at emails listed above. 
Acknowledgments: We would like to thank Beniamino De Santis and Simone Polini of Polini Distillates, Wines \& Beverages Group Italia s.r.l. for providing Sambuca and Mistrà samples and for helpful discussion of the data, and the enologist Gianni Branca of EVER s.r.l. for providing the light 7 stress apparatus for the determination of VIS light stress.

Conflicts of Interest: The authors declare no conflict of interest.

\section{References}

1. Carteau, D.; Brunerie, P.; Guillemat, B.; Bassani, D.M. Photochemistry in everyday life: The effect of spontaneous emulsification on the photochemistry of Trans-anethole. Photochem. Photobiol. Sci. 2007, 6, 423-430. [CrossRef]

2. Castro, H.T.; Martínez, J.R.; Stashenko, E. Anethole isomerization and dimerization induced by acid sites or UV irradiation. Molecules 2010, 15, 5012-5030. [CrossRef]

3. Zabetakis, I. Anise spirits: Types, sensory properties and sensory analysis. In Alcoholic Beverages: Sensory Evaluation and Consumer Research; Piggott, J., Ed.; Woodhead Publishing Limited: Cambridge, UK, 2012; pp. 229-241.

4. Sitnikova, N.L.; Sprik, R.; Wegdam, G.; Eiser, E. Spontaneously formed trans-anethol/water/alcohol emulsions: Mechanism of formation and stability. Langmuir 2005, 21, 7083-7089. [CrossRef] [PubMed]

5. Grillo, I. Small-angle neutron scattering study of a world-wide known emulsion: Le pastis. Colloids Surf. A Physicochem. Eng. Asp. 2003, 225, 153-160. [CrossRef]

6. Dawidar, A.M.; Abou-Elzahab, M.M.; Abdel-Mogib, M.; Hussien, K.; Mostafa, M.E.-H. Photo-oxygenation of trans anethole. Int. J. Sci. Eng. Appl. 2015, 4, 294-298. [CrossRef]

7. Lewis, F.D.; Kojima, M. Electron transfer induced photoisomerization, dimerization, and oxygenation of trans- and cis-anethole. The role of monomer and dimer cation radicals. J. Am. Chem. Soc. 1988, 110, 8664-8670. [CrossRef]

8. Aprotosoaie, A.C.; Costache, I.I.; Miron, A. Anethole and its role in chronic diseases. In Drug Discovery from Mother Nature. Advances in Experimental Medicine and Biology; Gupta, S.C., Prasad, S., Aggarwal, B.B., Eds.; Springer International Publishing: Basel, Switzerland, 2016; pp. 247-267. ISBN 1420026399.

9. Akçan, R.; Lale, A.; Tümer, A.R. Trans-anethole: A key compound in Bogma Raki. Acta Med. 2018, 49, $26-31$.

10. Mohan, R.S.; Whalen, D.L. Acid-catalyzed hydrolysis of cis- and trans-anethole oxides: Discrete carbocation intermediates and syn/anti hydration ratios. J. Org. Chem. 1993, 58, 2663-2669. [CrossRef]

11. O'Brien, P.; Siraki, A.; Shangari, N. Aldehyde sources, metabolism, molecular toxicity mechanisms, and possible effects on human health. Crit. Rev. Toxicol. 2005, 35, 609-662. [CrossRef]

12. Özgüven, M. Aniseed. In Handbook of Herbs and Spices; Peter, K.V., Ed.; Taylor \& Francis: London, UK, 2012; pp. 138-150. ISBN 9780857095688.

13. Zafeiropoulou, T.; Evageliou, V.; Gardeli, C.; Yanniotis, S.; Komaitis, M. Retention of trans-anethole by gelatine and starch matrices. Food Chem. 2010, 123, 364-368. [CrossRef]

14. Kfoury, M.; Auezova, L.; Greige-Gerges, H.; Ruellan, S.; Fourmentin, S. Cyclodextrin, an efficient tool for trans-anethole encapsulation: Chromatographic, spectroscopic, thermal and structural studies. Food Chem. 2014, 164, 454-461. [CrossRef] [PubMed]

15. Roberts, D.D.; Elmore, J.S.; Langley, K.R.; Bakker, J. Effects of sucrose, guar gum, and carboxymethylcellulose on the release of volatile flavor compounds under dynamic conditions. J. Agric. Food Chem. 1996, 44, 1321-1326. [CrossRef]

16. Misharina, T.A.; Polshkov, A.N. Antioxidant properties of essential oils: Autoxidation of essential oils from laurel and fennel and effects of mixing with essential oil from coriander. Appl. Biochem. Microbiol. 2005, 41, 610-618. [CrossRef]

17. Miething, H.; Seger, V.; Hänsel, R. Determination of photoanethole from a stored essential oil of anise fruits as $4,4^{\prime}$ dimethoxystilbene by high performance liquid chromatography-ultraviolet coupling. Phyther. Res. 1990, 4, 121-123. [CrossRef]

18. Turek, C.; Stintzing, F.C. Stability of essential oils: A review. C. R. Food Sci. Food Saf. 2013, 12, 40-53. [CrossRef]

19. Btaun, M.; Franz, G. Quality criteria of bitter fennel oil in the german pharmacopoeia. Pharm. Pharmacol. Lett. 1999, 9, 48-51.

20. Regulation (EU) 2019/787. Off. J. Eur. Union 2019, L 130, 1-54.

21. Jurado, J.M.; Alcázar, A.; Pablos, F.; Martín, M.J. LC Determination of anethole in aniseed drinks. Chromatographia 2006, 64, 223-226. [CrossRef]

22. Regulation (EC) No 2091/2002. Off. J. Eur. Communities 2002, L 322, 11-27.

23. Moretón-Lamas, E.; Lago-Crespo, M.; Lage-Yusty, M.A.; López-Hernández, J. Comparison of methods for analysis of resveratrol in dietary vegetable supplements. Food Chem. 2017, 224, 219-223. [CrossRef] [PubMed]

24. Magnusson, B.; Örnemark, U. (Eds.) Eurachem Guide: The Fitness for Purpose of Analytical Methods—A Laboratory Guide to Method Validation and Related Topics, 2nd ed.; EURACHEM Working Group, LGC: Middlesex, UK, 2014; ISBN 0-94948926-12-0.

25. Maury, C.; Clark, A.C.; Scollary, G.R. Determination of the impact of bottle colour and phenolic concentration on pigment development in white wine stored under external conditions. Anal. Chim. Acta 2010, 660, 81-86. [CrossRef] [PubMed]

26. Lewis, F.D.; Kojimat, M. Photodimerization of singlet trans- and cis-anethole. Concerted or stepwise? J. Am. Chem. Soc. 1988, 110, 8660-8664. [CrossRef]

27. Lukes, P.; Clupek, M.; Babicky, V.; Sunka, P. Ultraviolet radiation from the pulsed corona discharge in water. Plasma Sources Sci. Technol. 2008, 17, 024012. [CrossRef] 
28. Hennemeyer, M.; Burghardt, S.; Stark, R.W. Cantilever micro-rheometer for the characterization of sugar solutions. Sensors 2008, 8, 10-22. [CrossRef] [PubMed]

29. Alvarez, H.M.; Barbosa, D.P.; Fricks, A.T.; Aranda, D.A.G.; Valdés, R.H.; Antunes, O.A.C. Production of piperonal, vanillin, and $p$-anisaldehyde via solventless supported iodobenzene diacetate oxidation of isosafrol, isoeugenol, and anethol under microwave irradiation. Org. Process. Res. Dev. 2006, 10, 941-943. [CrossRef] 